CRÍTICA, Revista Hispanoamericana de Filosofía. Vol. 33, No. 99 (diciembre 2001): 47-73

\title{
SOBRE LA LÓGICA DE LAS LAGUNAS EN EL DERECHO*
}

José Juan Moreso

Universidad Pompeu Fabra josejuan.moreso@dret.upf.es

Pablo E. Navarro

CONICET/Universidad de Buenos Aires pnavarro@arnet.com.ar

María Cristina Redondo

CONICET/Universidad Nacional de Córdoba criredon@giuri.unige.it

RESUMEN: En "Legal Reasons, Sources and Gaps", Raz señala que las lagunas jurídicas existen sólo cuando el derecho habla con voz incierta o cuando habla con muchas voces, pero que no hay lagunas cuando el derecho guarda silencio. En este último caso habría reglas de clausura, analíticamente verdaderas, que impiden la ocurrencia de esas lagunas.

Según Raz, si hay una laguna en un sistema jurídico, entonces no es verdadero ni falso que exista una razón concluyente para ejecutar cierta acción. Así, una de las contribuciones más importantes de Raz a la solución del problema de las lagunas jurídicas es subrayar que el discurso jurídico no está completamente controlado por la bivalencia. Sin embargo, a menudo se sostiene que el rechazo de la bivalencia conduce a contradicciones. Si esta afirmación fuese verdadera, entonces la solución de Raz al problema de las lagunas jurídicas se vería seriamente amenazada.

En este artículo mostramos, con ayuda de una herramienta analítica sofisticada, i.e., la lógica de la verdad de G.H. von Wright, que el rechazo de la bivalencia sólo nos compromete a aceptar la conclusión trivial de que puede haber proposiciones que no son verdaderas ni falsas. Por esta razón, el trabajo de Raz todavía puede ser considerado un buen punto de partida para analizar las relaciones entre normas, razonamiento práctico y lagunas jurídicas. Sin embargo, también mostramos que para admitir proposiciones que no son

* Los autores desean agradecer al comité de dirección de Crítica y a dos evaluadores anónimos las numerosas observaciones y sugerencias hechas a una versión anterior de este artículo y a la que se publica en este volumen. En particular, a lo largo de casi dos años, las discusiones con los evaluadores nos han permitido advertir defectos formales y sustanciales de nuestro trabajo. Aunque no siempre hemos coincidido con las objeciones de los evaluadores, queremos mencionar nuestro reconocimiento a sus preocupaciones por mejorar la calidad de nuestro artículo. 
verdaderas ni falsas, las tesis de Raz deben ser reformuladas. De otra manera, la tesis de que no existen lagunas cuando el derecho guarda silencio no sería compatible con el rechazo de la bivalencia.

PALABRAS CLAVE: lagunas jurídicas, bivalencia, razones concluyentes, lógica de la verdad

SUMMARY: In his paper "Legal Reasons, Sources and Gaps", Raz says that legal gaps only exist when law speaks with uncertain voice or when it speaks with many voices, but there are no gaps when law is silent. In this later case, rules of closure, which are analytically true, prevent from the occurrence of gaps.

According to Raz, if there is a gap in a legal system, then both the claim that there is a conclusive legal reason to perform a certain action, and its negation are neither true nor false. Therefore, one of the Raz's most important contributions to the solution of the problem of legal gaps is to remark that legal discourse is not altogether governed by the principle of bivalence. However, philosophers often claim that the denial of bivalence leads to a logical inconsistency. If this claim were true, then Raz's solution to the problem of gaps would be seriously threatened.

In this paper we show - with the aid of a sophisticated analytical tool, i.e., von Wright's truth-logic - that the rejection of bivalence only commits us to accept the trivial conclusion that propositions can lack truth-values. For this reason, Raz's paper can still be regarded as a good starting-point for analyzing the relationships between norms, practical reasoning and legal gaps. However, we also show that in order to admit propositions which are neither true nor false, Raz's theses must be reformulated. Otherwise, the claim that there is no gap when law is silent would not be compatible with the rejection of bivalence.

KEY WORDS: legal gaps, bivalence, conclusive reasons, truth-logic

\section{Introducción}

En su influyente artículo "Legal Reasons, Sources and Gaps", Joseph Raz ha dedicado considerables esfuerzos a clarificar el problema de las lagunas en el derecho. ${ }^{1}$ En los trabajos clásicos sobre este problema en filosofía del derecho, las lagunas del derecho se vinculan principalmente a la inexistencia de normas en un sistema jurídico. ${ }^{2}$ En parte, la novedad del enfoque de Raz consiste en apartarse de esta estrategia tradicional. En este sentido, Raz vincula el problema de las lagunas a la posibilidad de determinar el valor de verdad de cierto tipo de enunciados jurídicos, i.e., enunciados acerca de las

1 J. Raz, "Legal Reasons, Sources and Gaps", reimpreso en The Authority of Law, pp. 53-77. En adelante se citará como LSG.

${ }^{2}$ Véase, por ejemplo, Alchourrón y Bulygin 1971. 
razones concluyentes que el derecho ofrece para ejecutar determinadas acciones. ${ }^{3}$ Las razones jurídicas concluyentes son soluciones o respuestas que el derecho ofrece a ciertos problemas prácticos; por ejemplo, cuando un individuo $x$ tiene que decidir acerca de la ejecución de una acción $\phi$. De esta manera, las lagunas en el derecho se manifiestan en la ausencia de una respuesta completa acerca de lo que cierto individuo $x$ debe hacer jurídicamente en determinado momento. Raz sostiene que hay una respuesta jurídica completa cuando: ${ }^{4}$ (a) es verdad que el derecho requiere concluyentemente la acción $\phi$ a un sujeto $x$. Esta situación se representa como " $L R_{c} x$, $\phi$ ", o (b) es verdad que el derecho permite concluyentemente a $x$ no hacer $\phi$. Raz representa esta situación mediante la fórmula $\operatorname{LPer}_{c} x, \neg \phi, y$ también sostiene que es equivalente a $L \neg R_{c} x, \phi .^{5}$

Según Raz, la relación entre respuestas completas y lagunas jurídicas se puede caracterizar de la siguiente manera:

Un sistema jurídico es jurídicamente completo si existe una respuesta completa a todas las cuestiones jurídicas sobre las que los tribunales tienen jurisdicción. Un sistema jurídico contiene lagunas si algunas cuestiones jurídicas sobre las que los tribunales tienen jurisdicción no tienen respuesta completa. ${ }^{6}$

De la primera afirmación de esta cita se sigue que si hay respuestas completas $(R C)$, entonces no existen lagunas jurídicas $(L G)$ (i.e. el sistema jurídico es completo). Es decir,

(a) $R C \rightarrow \neg L G$

3 LSG, pp. 62-68.

4 LSG, p. 71.

${ }^{5}$ LSG, p. 76.

${ }^{6}$ LSG, p. 70. Es posible que la calificación de una determinada acción resulte indeterminada debido a que los jueces carecen de jurisdicción para decidir en cierto tipo de situaciones. Ello constituiría una laguna jurisdiccional. La naturaleza y el alcance de este tipo de lagunas no serán considerados en este trabajo. Al igual que Raz, en nuestro análisis de las lagunas jurídicas asumiremos que los jueces tienen jurisdicción para decidir acerca de todas las situaciones reguladas por el derecho. 
De su segunda afirmación se sigue que si no hay respuestas completas, entonces hay lagunas; es decir,

(b) $\neg R C \rightarrow L G$

Por contraposición, se sigue de (b) que

(c) $\neg L G \rightarrow R C$

Es fácil advertir, entonces, que de (a) y (c) se sigue

(d) $R C \leftrightarrow \neg L G$

En otras palabras, conforme al esquema conceptual propuesto por Raz, no existen lagunas jurídicas acerca de la calificación de cierta acción si y sólo si existen respuestas completas para esa acción.

Como hemos visto anteriormente, con respecto a la acción $\phi$ y a un sujeto $x$, existe una respuesta completa cuando es verdad que el derecho requiere concluyentemente $\phi$ a $x$ (en símbolos: " $\left.L R_{c} x, \phi "\right)$ o bien cuando es verdad que el derecho no requiere concluyentemente $\phi$ a un sujeto $x$ (en símbolos: " $\neg L R_{c} x, \phi$ "). Según Raz, $\neg L R_{c} x, \phi$ es equivalente a " $L \neg R_{c} x, \phi$ ", y esta equivalencia desempeña un papel central en sus argumentos acerca de la existencia de lagunas jurídicas. Más adelante analizaremos en detalle esta equivalencia y las consecuencias que de ella se siguen. A efectos de simplificar el simbolismo de Raz presupondremos que siempre nos referimos a una razón concluyente $\phi$ relativa a un sujeto $x$. De este modo, la fórmula " $L R_{c} x, \phi$ " se representará simplemente como " $L R$ ", y " $L \neg R$ " reemplazará a " $L \neg R_{c} x, \phi "$.

Del enfoque de Raz se deriva que la existencia de lagunas jurídicas es incompatible con la verdad de la siguiente disyunción:

(0) $L R \vee L \neg R$

En adelante, (0) se denominará la tesis de las respuestas completas y representará un criterio de adecuación del concepto de laguna jurídica; es decir, la existencia de una laguna jurídica implica que la tesis de las respuestas completas no es verdadera y la verdad de la tesis de las respuestas completas implica la inexistencia de lagunas. 
Según Raz, hay dos clases posibles de lagunas jurídicas. El primer tipo de lagunas, que denominaremos lagunas ordinarias, es una negación heterodoxa de la tesis (0). Las lagunas del segundo tipo, que denominaremos lagunas genuinas, son una negación clásica de $(0){ }^{7}$

(1) Lagunas ordinarias. La proposición " $L R$ " no es verdadera ni falsa y la proposición " $L \neg R$ " no es verdadera ni falsa; y

(2) Lagunas genuinas. La conjunción " $\neg L R \& \neg L \neg R$ ” es verdadera.

Las lagunas ordinarias surgen como consecuencia de la vaguedad de los conceptos jurídicos o por conflictos normativos sin solución en el sistema. En estos casos, el derecho se expresa con "voz incierta" o con "demasiadas voces", y por ello no determina concluyentemente lo que debemos hacer. A su vez, las lagunas genuinas se presentarían cuando el derecho no regula la acción en cuestión. En casos de lagunas genuinas, el derecho "guarda silencio". Aunque Raz introduce en su análisis ingredientes extraños a la lógica proposicional clásica $(L P)$, e.g., proposiciones que no son verdaderas ni falsas, sus demostraciones y argumentos se representan mediante operaciones proposicionales clásicas. En $L P$, la negación de una proposición $p$ está asociada a la falsedad de $p$. Así, se puede señalar que las lagunas genuinas se presentarían sólo cuando la tesis de las respuestas completas es falsa; i.e. (2) es equivalente a la falsedad de la tesis de las respuestas completas:

(3) $\neg(L R \vee L \neg R)$.

Por tanto, sólo si (2) — y por lo tanto también (3) — fuese(n) verdadera(s), serían posibles las genuinas lagunas normativas. ${ }^{8}$ Pero, según Raz, es lógicamente verdadero que no existe una razón jurídica concluyente para $\phi$ si y sólo si según el derecho no se requiere concluyentemente $\phi$. Así, según Raz es analíticamente verdadero que: ${ }^{9}$

\footnotetext{
7 LSG, p. 71.

8 LSG, p. 76.

9 LSG, p. 76.
} 
(4) $\neg L R \leftrightarrow L \neg R$.

De (2) y (4) se sigue:

(5) $L \neg R \& \neg L \neg R$. $^{10}$

Sin embargo, (5) es una contradicción, y por consiguiente la verdad lógica expresada por (4) implica el rechazo de (2) y (3). En otras palabras, (4) implica el rechazo de la existencia de lagunas jurídicas genuinas. (4) también implica

(6) $(\neg L R) \rightarrow\left(L P e r_{c} x, \neg \phi\right)$

Por ello, Raz añade:11 "Esto nos conduce a la conocida regla de clausura de acuerdo con la cual lo que no está jurídicamente prohibido, está jurídicamente permitido."

El núcleo del argumento de Raz radica en la tesis (4). Raz afirma que (4) es una verdad lógica, y defiende su tesis de la siguiente manera. Primero ofrece una prueba para $L \neg R \rightarrow \neg L R$. Para ello afirma que no puede ser verdad que jurídicamente haya una razón concluyente para realizar un acto $(i . e . L R) \mathrm{y}$, al mismo tiempo, que jurídicamente no exista esa razón $($ i.e. $L \neg R){ }^{12}$

Entonces, hay que admitir (7) $L R \rightarrow \neg L \neg R$, y por contraposición se deriva

(8) $L \neg R \rightarrow \neg L R$.

Luego, Raz prueba (9) $\neg L R \rightarrow L \neg R$. Para ello introduce el siguiente argumento: ${ }^{13}$

Asumamos que el antecedente es verdadero, i.e., $L R_{c} x, \phi$ es falso. ¿Puede ser falso también $L \neg R_{c} x, \phi$ ? $\mathrm{Si} \neg L R_{c} x, \phi$, entonces no es el caso que haya un hecho que sea una razón jurídicamente concluyente para $\phi$. Pero, si no hay ninguna razón jurídica concluyente

${ }^{10}$ La prueba de (5) es sencilla:
[a] $\neg L R$
[b] $L \neg R$
[c] $\neg L \neg R$
Eliminación de \& en (2).
[d] $L \neg R \& \neg L \neg R$
Modus ponens en (4) y [a].
Eliminación de \& en (2).
Introducción de \& en [b], [c].

11 LSG, p. 76.

12 LSG, p. 76.

${ }^{13}$ LSG, p. 76. 
para $\phi$, ¿podría dejar de ser que, conforme al derecho, no haya ninguna razón concluyente para $\phi$ ? Ésta habría sido una posibilidad real si se hubiese admitido la necesidad de un tipo especial de hechos para hacer verdaderos los enunciados negativos de razones de la forma $\neg R_{c} x, \phi \ldots$ Tales enunciados se verifican únicamente por la ausencia de razones, y es precisamente tal ausencia la que está implicada en $\neg L R_{c} x, \phi$. De allí que $\neg L R_{c} x, \phi \rightarrow L \neg R_{c} x, \phi$. Q.E.D.

En resumen, Raz niega la existencia de lagunas genuinas en el derecho. De este modo, cuando el derecho "guarda silencio", operan reglas de clausura que resuelven las situaciones de indeterminación. En contraste, Raz señala que las lagunas ordinarias son inevitables. En esos casos, cuando el derecho se expresa "con voz incierta" o "con diferentes voces" no existe una única respuesta jurídica correcta, y sería inevitable cierto margen de discreción judicial para superar la indeterminación.

\section{Objetivos y estructura del trabajo}

El enfoque de Raz ha ejercido una considerable influencia en la comunidad filosófica. ${ }^{14}$ Sin embargo, sus tesis acerca de la indeterminación del derecho presentan una "tensión conceptual" que merece un análisis lógico más cuidadoso. El objetivo principal de este trabajo es considerar si es posible rechazar las lagunas genuinas y — al mismo tiempo - admitir la existencia de lagunas ordinarias. En nuestra discusión presupondremos que Raz tiene razón cuando sostiene que la tesis (4) — que se denominará la ley de $R a z$ - es una verdad analítica. ${ }^{15}$ Aunque Raz no considera explícitamente si la validez de la tesis (4) afecta a las lagunas

14 Véanse, por ejemplo, Gardner 1988, pp. 457-461; Bix 1993, pp. 25-26; Shiner 1992, pp. 189-191; Eisenberg 1988, pp. 146-161.

15 La versión del principio de clausura que sostiene Raz se sigue de la noción misma de permisión concluyente. Un permiso concluyente para una determinada acción es incompatible con una razón concluyente para omitir esa acción. Por ello, cualquier acción, e.g. $\phi$, está concluyentemente determinada en un sistema jurídico ya que ello sólo significa que $\phi$ está prohibida por una razón concluyente, o bien que $\phi$ no está prohibida por una razón concluyente. Véase LSG, p. 64. 
ordinarias, la existencia de estas lagunas es dudosa ya que la ley de Raz también implica la tesis de las respuestas completas, i.e., la tesis $(0) .{ }^{16}$

Más aún, un vínculo entre (4) y el principio de bivalencia se insinúa claramente en la reconstrucción de Raz, ya que la siguiente tesis es también una consecuencia lógica de la ley de Raz:

(10) $L R \vee \neg L R$

La tesis (10) $L R \vee \neg L R$, que se sigue de (0) y la ley de Raz, puede ser considerada una instancia del principio de bivalencia. Por ello, dado que (4) nos compromete a aceptar tanto la tesis de las respuestas completas como, presumiblemente, una versión del principio de bivalencia, es razonable preguntarse si existe algún espacio conceptual para proposiciones que no son verdaderas ni falsas.

En otras palabras: las lagunas genuinas son refutadas por (4) $\neg L R \leftrightarrow L \neg R$. Sin embargo, dado que (4) también implica la tesis de las respuestas jurídicas completas, i.e. (0), hay tensión interna entre las lagunas ordinarias y la ley de Raz. Esta tensión se oculta parcialmente en el simbolismo de Raz porque no pueden representarse aquellas proposiciones que carecen de valor de verdad. En particular, la negación de " $L R$ " es ambigua y puede significar que " $L R$ " es falsa, o bien simplemente que no es verdadera. ${ }^{17}$ Por esta razón es interesante analizar si las intuiciones de Raz se pueden preservar en una reconstrucción lógica más cuidadosa, y de esta manera determinar de qué forma puede resolverse la tensión entre las lagunas ordinarias y la ley de Raz.

16 Prueba:
a) $\neg L R \leftrightarrow L \neg R$
b) $\neg L R \rightarrow L \neg R$
c) $L R \vee L \neg R$
Ley de Raz, i.e. 4
Eliminación en a
PL

17 Raz dice que " $\neg L R$ " significa "no es el caso que jurídicamente se deba $\phi "$ (LSG, p. 67). No obstante, la ambigüedad se mantiene ya que esta lectura es compatible no sólo con la falsedad de " $L R$ " sino también con una afirmación más débil, i.e., " $L R$ " no es verdadera. 
En la sección 3 introduciremos una herramienta conceptual, i.e., el cálculo truth-logic $(T L)$ elaborado por von Wright en diversos ensayos, que nos permitirá superar las deficiencias del simbolismo de Raz. En particular, mediante $T L$ abordaremos el problema de aquellas proposiciones que no son verdaderas ni falsas. En la sección 4 examinaremos si las importantes intuiciones de Raz acerca de las lagunas jurídicas ordinarias se pueden preservar en $T L$. En la sección 5 analizaremos dos posibles interpretaciones de la ley de Raz en $T L$, y finalmente, en 6, estudiaremos algunas consecuencias del rechazo de la bivalencia en $T L$.

\section{Una lógica de la verdad}

En "Demystifying Propositions", Georg Henrik von Wright ofrece una sugerente propuesta conceptual para caracterizar las proposiciones. ${ }^{18}$ La noción básica de la propuesta de von Wright es la de enunciado bien formado de un lenguaje $L$. Los criterios para determinar cuáles son los enunciados bien formados de un lenguaje $L$ son reconstruidos por los lingüistas y — prima facieno son objeto de polémica filosófica.

Un enunciado bien formado $E$ del lenguaje $L$, por ejemplo, "La nieve es blanca", expresa una proposición si y sólo si, al añadirse el prefijo "Es verdad que" (en adelante prefijo $T$, o simplemente $T$ ) al enunciado $E$, se obtiene otro enunciado bien formado $E_{1}$ del lenguaje $L$. Por tanto, afirmar que un enunciado expresa una proposición sólo significa que, en el lenguaje $L$, es posible obtener un enunciado $E_{1}$ al añadir el prefijo $T$ a $E$.

Conforme a esta reconstrucción, un enunciado puede expresar una proposición incluso cuando tengamos discrepancias acerca de si es posible atribuirle valor de verdad. Así, los enunciados "El rey de Francia es calvo" o "los números primos son azules" expresan una proposición con independencia de la posición que tomemos acerca de su valor de verdad.

${ }^{18}$ Von Wright 1984a, pp. 14-25. 
En su artículo "Truth and Logic", ${ }^{19}$ von Wright construye un cálculo al que denomina "lógica de la verdad" (truth-logic: $T L$ ), el cual permite analizar con claridad algunas consecuencias conceptuales importantes de su enfoque acerca de las proposiciones. El vocabulario básico de $T L$ es el siguiente:

a) Variables $p, q, \ldots$, que representan enunciados declarativos, i.e., enunciados que admiten su transformación mediante una "que-cláusula".

b) Dos conectivas: " $\neg$ " para la negación, que corresponde a la palabra "no" de los lenguajes naturales, y "\&" para la conjunción, que corresponde a la palabra "y" de los lenguajes naturales.

c) Un operador $T$, que se lee "Es verdad que".

d) Paréntesis.

Otras conectivas, correspondientes a la disyunción, el condicional y el bicondicional, se introducen mediante las siguientes definiciones:

$$
\begin{aligned}
& S \vee S^{\prime}=d f \neg\left(\neg S \& \neg S^{\prime}\right) \\
& S \rightarrow S^{\prime}=d f \neg\left(S \& \neg S^{\prime}\right) \\
& S \leftrightarrow S^{\prime}=d f \neg\left(S \& \neg S^{\prime}\right) \& \neg\left(\neg S \& S^{\prime}\right)
\end{aligned}
$$

Las expresiones bien formadas del cálculo son $T$-fórmulas. Las $T$-fórmulas se pueden definir recursivamente de la siguiente manera:

(i) Las $T$-fórmulas son atómicas o moleculares.

(ii) Una fórmula $T$-atómica consiste en el operador $T$ seguido por:

a) una variable $(e . g . T p)$, o

b) por un compuesto molecular de variables (e.g. $T(p \&$ q)), o

c) por una fórmula $T$-atómica $(e . g . T T \neg p), o$

d) por un compuesto de $T$-fórmulas atómicas (e.g. $T(T p \&$ $T q)$ ), o

19 Von Wright 1984c, pp. 26-4l. 
e) por una variable o compuesto molecular de variables y fórmulas $T$-atómicas $(e . g . T(p \rightarrow T q))$.

(iii) Una fórmula $T$-molecular es un compuesto molecular de $T$-fórmulas atómicas $(e . g . T p \rightarrow T \neg T q) .^{20}$

Las "bases" del cálculo $T L$ son las siguientes:

(A1) $T \neg p \rightarrow \neg T p$

(A2) $T p \leftrightarrow T \neg \neg p$

(A3) $T(p \& q) \leftrightarrow T p \& T q$

(A4) $T \neg(p \& q) \leftrightarrow T \neg p \vee T \neg q$

(A5) $T \neg T p \leftrightarrow \neg T p$

La clase de teoremas de $T L$ se define de la siguiente manera:

(T1) Una $T$-fórmula que se obtiene a partir de una tautología de $L P$ prefijando la letra $T$ inmediatamente enfrente de cada variable de la tautología de $L P$. Así, si $\neg p \vee p$ es una tautología de $L P$, entonces $\neg T p \vee T p$ es un teorema de $T L .^{21}$ En general, la sustitución de las variables proposicionales en una tautología por $T$-fórmulas es un teorema de $T L$.

(T2) Los axiomas A1-A5 son teoremas de $T L$.

(T3) $T$-fórmulas que pueden ser obtenidas a partir de teoremas de $T L$ con la ayuda de las siguientes reglas:

R1 Sustitución de variables por otras variables, por un compuesto molecular de variables o por $T$-fórmulas.

R2 Regla de separación o modus ponens.

R3 Regla de la verdad. Si $s$ es un teorema, Ts también es un teorema.

Cuatro metateoremas son especialmente importantes en $T L:^{22}$

20 Las fórmulas mixtas, $e . g ., p \rightarrow T p$ no son admitidas en esta presentación de $T L$; sin embargo, en su última presentación de $T L$, von Wright abandona esta restricción. Véase von Wright 1996, pp. 71-91.

21 Él incorpora esta cláusula al núcleo axiomático en von Wright 1996. Véase la p. 74.

22 Von Wright 1984c, pp. 28-31. 
(M1) Si $S \leftrightarrow S^{\prime}$ es un teorema de $T L$, entonces $S$ y $S^{\prime}$ son intercambiables salva veritate en las $T$-fórmulas.

(M2) Una $T$-fórmula es equivalente a una $T$-fórmula de primer orden, i.e., una fórmula en la que ninguna $T$ está dentro del alcance de otra $T$.

(M3) Una $T$-fórmula es equivalente a un compuesto molecular de fórmulas $T$-atómicas de la forma simple de una $T$ seguida ya sea por una única variable o por la negación de una única variable. Estas fórmulas se denominan "constituyentes de verdad" o " $T$-constituyentes" de la fórmula $T$ original.

(M4) Todos los teoremas de $T L$ son $T$-tautologías y todas las $T$ tautologías son teoremas de $T L$. La noción de $T$-tautología es introducida por von Wright de la siguiente manera: ${ }^{23}$

Dado que una fórmula- $T$ es equivalente a un compuesto molecular de sus $T$-constituyentes, puede usarse una tabla de verdad para investigar qué función de verdad de los constituyentes expresa esa fórmula. Si expresa una tautología, denominaremos a la fórmula (o a la proposición que ella expresa) una verdadera tautología o $T$-tautología.

Para von Wright, la proposición que afirma "Es verdad que $p "(e . g$. " $T p ")$ puede ser negada de dos maneras diferentes. Por una parte, la negación externa de esta proposición es "No es verdad que $p$ ", es decir, " $\neg T p$ ", y su comportamiento lógico es similar a la negación clásica ya que es exhaustiva y excluyente. Por otra parte, la negación interna es "Es verdad que $\neg p$ ". Dado que la falsedad de una proposición $p$ puede ser definida como la verdad de su negación, i.e., afirmar que es verdadera la negación de $p$, es decir $T \neg p$, puede leerse como "es falso que $p$ ".

El axioma Al señala que si una proposición es falsa, entonces no es verdadera. Pero en $T L$ no se puede probar que si una proposición no es verdadera entonces es falsa. Esto abre un espacio conceptual para dar cuenta de aquellas proposiciones que no son verdaderas ni falsas. Por consiguiente, si afirmamos que

${ }^{23}$ Von Wright 1984c, p. 31. 
una proposición $p$ es falsa (e.g., " $T \neg p$ '), entonces estamos comprometidos a aceptar que $p$ no es verdadera (e.g., " $\neg T p$ "). Pero, dado que una proposición puede carecer de valor de verdad, no puede admitirse que " $\neg T p$ " implica " $T \neg p$ ".

La decisión acerca del valor de verdad de las fórmulas de $T L$ se puede tomar conforme a las siguientes tablas de verdad. En estas tablas, el valor "verdad" está representado por "V", el valor "falso" por "F", y el valor "ni verdadero ni falso" por el símbolo "?".

\begin{tabular}{cccccc}
$p$ & $\neg p$ & $T p$ & $T \neg p$ & $\neg T p$ & $\neg T \neg p$ \\
\hline $\mathrm{V}$ & $\mathrm{F}$ & $\mathrm{V}$ & $\mathrm{F}$ & $\mathrm{F}$ & $\mathrm{V}$ \\
$\mathrm{F}$ & $\mathrm{V}$ & $\mathrm{F}$ & $\mathrm{V}$ & $\mathrm{V}$ & $\mathrm{F}$ \\
$?$ & $?$ & $\mathrm{~F}$ & $\mathrm{~F}$ & $\mathrm{~V}$ & $\mathrm{~V}$
\end{tabular}

Conforme a este método de decisión, el valor de verdad de una $T$-fórmula depende del valor de verdad asignado a una variable proposicional. Sin embargo, aunque podemos asignar "?" a una variable, una $T$-fórmula siempre será verdadera o falsa. En $T L$, las tablas para decidir el valor de verdad de otras fórmulas se elaboran a partir de las siguientes asignaciones:

a) Una conjunción es verdadera cuando todos sus miembros son verdaderos. Una conjunción es falsa cuando al menos uno de sus miembros es falso. En los casos restantes, la conjunción es indeterminada, i.e., recibe el valor "?".

b) Una disyunción es verdadera cuando uno de sus miembros es verdadero. La disyunción es falsa cuando todos sus miembros son falsos. En los casos restantes, la disyunción es indeterminada, i.e., recibe el valor "?".

c) Un condicional es verdadero cuando su antecedente es falso o su consecuente es verdadero. El condicional es falso cuando su antecedente es verdadero y su consecuente falso. En los casos restantes, el condicional es indeterminado, i.e., recibe el valor "??".

d) Un bicondicional es verdadero cuando sus miembros son ambos verdaderos o son ambos falsos. El bicondicional es falso cuando uno de sus miembros es verdadero y el otro 
falso. En los casos restantes, el bicondicional es indeterminado, i.e., recibe el valor "?".

Es importante destacar tres características centrales de $T L$ : (a) el alcance restringido de la bivalencia, (b) el "espacio conceptual" para representar aquellas proposiciones que no son verdaderas ni falsas, y (c) las diferencias entre tautologías de $L P$ y las tautologías de $T L$.

(a) Alcance restringido de la bivalencia y tercero excluido. En $T L$ debemos distinguir el principio de bivalencia, que afirma que toda proposición es verdadera o falsa, e.g., $T p \vee T \neg p$, de la ley del tercero excluido, según la cual una proposición $p$ es verdadera o no es verdadera, e.g., $T p \vee \neg T p$. Mientras que $T p \vee \neg T p$ es una tautología en $T L, T p \vee T \neg p$ no puede ser probada en $T L$. Sin embargo, el principio de bivalencia puede ser "recuperado" en $T L$ de otra manera diferente:

Alcance restringido de la bivalencia clásica: es verdad que $p$ o no- $p$ si y sólo si es verdad que $p$ o es falso que $p$, i.e., $T(p \vee \neg p) \leftrightarrow T p \vee T \neg p$.

Bivalencia en $T L$ : es verdad que $p$ es verdadera, o es falso que $p$ es verdadera, i.e., $T T p \vee T \neg T p$.

(b) Proposiciones que no son verdaderas ni falsas. Las $T$ fórmulas son siempre verdaderas o falsas. De este modo, aunque $T L$ tiene la capacidad expresiva suficiente para asignar un valor indeterminado, i.e. "?", a cierta proposición $p$, la afirmación de que $p$ no es verdadera ni falsa, i.e., $\neg T p \& \neg T \neg p$, es siempre verdadera o falsa. Precisamente, esta última fórmula es verdadera únicamente cuando $p$ recibe el valor "?". Es conveniente destacar que $T L$ no afirma que existen proposiciones que carecen de valor de verdad. Es decir, nada impide aplicar $T L$ a razonamientos cuyas premisas fuesen únicamente verdaderas o falsas. Por esta razón, hay que enfatizar que la fórmula $(\neg T p \& \neg T \neg p)$ $n o$ es un teorema de $T L$. No obstante, la afirmación más débil $(\neg T p \& \neg T \neg p) \leftrightarrow \neg(T p \vee T \neg p)$ es una tautología de $T L$. Esta última fórmula dice que una proposición no es verdadera ni falsa si y sólo si se niega la bivalencia. 
(c) Las tautologías en LP y en TL. Conforme a los criterios introducidos para reconocer una fórmula bien formada de $T L$, sólo son fórmulas de $T L$ aquellas expresiones $T$-atómicas o $T$ moleculares. De allí se sigue que ninguna expresión de $L P$ es una fórmula bien formada de $T L$. En particular, ninguna tautología de $L P$ es un teorema de $T L$, ya que esas expresiones de $L P$ no satisfacen los criterios para identificar $T$-fórmulas atómicas o moleculares. Ahora bien, afirmar que una tautología de $L P$, e.g. $(p \vee \neg p)$ es verdadera puede ser representada mediante, por ejemplo, la expresión $T(p \vee \neg p)$. Esta última expresión es una fórmula bien formada de $T L$. Sin embargo, $T(p \vee \neg p)$ no es un teorema de $T L$ ya que es falsa precisamente cuando $p$ carece de valor de verdad. Esto no significa que las tautologías de $L P$ sean por completo irrelevantes para $T L$. Por el contrario, según señala (T1), la sustitución de las variables proposicionales en una tautología por $T$-fórmulas es un teorema de $T L$. Así, aunque $(p \rightarrow p)$ no es válida en $T L$, la expresión $(T p \rightarrow T p)$ es un teorema de ese cálculo.

En resumen, $T L$ parece una herramienta adecuada para analizar aquellas proposiciones que no son verdaderas ni falsas. En la siguiente sección consideraremos si $T L$ puede contribuir al análisis de las lagunas en el derecho.

\section{Acerca de las lagunas ordinarias}

$T L$ proporciona una sofisticada herramienta analítica que nos permite representar proposiciones jurídicas que carecen de valor de verdad. Esto significa que, tal vez, $T L$ sea una herramienta adecuada para exponer las intuiciones de Raz de una manera más clara. Para alcanzar este objetivo, es preciso modificar previamente el vocabulario de $T L$ para dar cuenta de las fórmulas " $L R$ "; " $\neg L R$ "; " $L \neg R$ " y " $\neg L \neg R$ ". De esta manera, una $T$ fórmula podrá ahora también ser del tipo $T L R$. Al igual que las $T$-fórmulas, las $T L R$-fórmulas son atómicas o moleculares. Una fórmula $T L R$-atómica consiste en el operador $T$ seguido por la expresión $L R$ (e.g., $T L R)$, o un compuesto molecular de expresiones $L R(e . g ., T(L R \vee L \neg R)$, o por una fórmula $T L R$-atómica (e.g., $T T L R)$, o por un compuesto de $T$-fórmulas atómicas (e.g., 
$T(T L R \& T L \neg R)$ ), o por una variable o compuesto molecular de variables y fórmulas $T$-atómicas $(e . g ., T(p \rightarrow T L R))$. Una fórmula $T$-molecular es un compuesto molecular de $T$-fórmulas atómicas (e.g., $T L R \rightarrow T \neg T L R) .{ }^{24}$ Una vez que se extiende el vocabulario de $T L$ para dar cuenta de proposiciones acerca de razones jurídicas concluyentes, hay que destacar que ninguna de las fórmulas que utiliza Raz es una fórmula bien formada en $T L$. Por esta razón es preciso reformular sus tesis más importantes a efectos de analizar sus consecuencias en $T L$.

La tesis de las respuestas jurídicas completas — la tesis (0) que establece el criterio de adecuación de nuestros conceptos de laguna en el derecho será reformulada de la manera más intuitiva posible: simplemente prefijaremos el operador $T$ ante la tesis de las respuestas jurídicas completas. Así, (0) será representada como $\left(0^{\prime}\right) T(L R \vee L \neg R)$.

Dado que Raz afirma que existe una laguna ordinaria cuando ni " $L R$ " ni " $L \neg R$ " son verdaderas ni falsas, entonces las lagunas ordinarias podrían representarse como

(11) $\neg T L R \& \neg T \neg L R \& \neg T L \neg R \& \neg T \neg L \neg R$.

Las lagunas genuinas surgirían en la reconstrucción de Raz sólo si la tesis de las respuestas completas fuese falsa. De allí que, en TL, las lagunas genuinas podrían simbolizarse del siguiente modo: $T \neg(L R \vee L \neg R)$. Esta fórmula es equivalente a:

(12) $T(\neg L R \& \neg L \neg R)$

Ambas, (11) y (12), niegan que la tesis $\left(0^{\prime}\right)$ sea verdadera, aunque hay algunas diferencias importantes entre ellas; por ejemplo, (11) implica que $\left(0^{\prime}\right)$ no es verdadera, i.e., $\neg T(L R \vee L \neg R)$, pero (12) equivale a afirmar algo más fuerte, i.e., que $\left(0^{\prime}\right)$ es falsa, $T \neg(L R \vee L \neg R)$.

Es importante destacar que la llamada ley de Raz, $\neg L R \leftrightarrow$ $L \neg R$ no es una fórmula bien formada de $T L$. Dada la importancia de esta tesis en el argumento de Raz, es necesario considerar

${ }^{24}$ Debe advertirse que $L R$ y otras combinaciones veritativas obtenidas a partir de ella no son variables y, por ello, no pueden ser sustituidas mediante la regla Rl de $T L$. Agradecemos a un evaluador anónimo de Crítica esta corrección. 
una nueva formulación de esta tesis en $T L$. ¿Podría representarse la ley de Raz mediante la fórmula (13) $T(\neg L R \leftrightarrow L \neg R)$ ?

El siguiente argumento muestra que la respuesta es negativa. Uno de los objetivos básicos de Raz es señalar que, en cierta medida, el derecho siempre está indeterminado ya que es imposible eliminar completamente las lagunas ordinarias. Si existe una laguna ordinaria, entonces las proposiciones " $L R$ " y " $L \neg R$ " no son verdaderas ni falsas. Supongamos que representamos como " $\neg T L R \& \neg T \neg L R$ " la proposición que afirma que " $L R$ " no es verdadero ni falso. La fórmula " $\neg T L R \& \neg T \neg L R$ " es verdadera cuando existe una laguna ordinaria. Pero esta fórmula es incompatible con la nueva formulación de la ley de Raz, i.e., $T(\neg L R \leftrightarrow L \neg R)$.
(i) $T(\neg L R \leftrightarrow L \neg R)$
(ii) $\neg T L R \& \neg T \neg L R$
Nueva ley de Raz
(iii) $T(\neg L R \rightarrow L \neg R)$
Hipótesis
(iv) $T(L R \vee L \neg R)$
$L P$, i
(v) $T L R \vee T L \neg R$
$L P$, iii
(vi) $\neg T L R \rightarrow T L \neg R$
$\mathrm{A} 4, \mathrm{~A} 2^{25}$
(vii) $\neg(\neg T L R \rightarrow T \neg L R)$
$L P, \mathrm{v}$
$\begin{array}{lll}\text { (vii) } \neg(\neg T L R \rightarrow T \neg L R) & L P, \text { ii } \\ \text { (viii) }(\neg T L R \rightarrow T L \neg R) \& \neg(\neg T L R \rightarrow & \text { vi, vii }\end{array}$ $T \neg L R)$

La conclusión (vii) es una contradicción en TL. Por consiguiente, la propuesta de Raz se encontraría nuevamente ante la amenaza de incoherencia, ya que admite que la nueva formulación de su tesis (4), i.e. la ley de Raz, lleva a una incoherencia en aquellos casos en que las proposiciones " $L R$ " y " $L \neg R$ " no son verdaderas ni falsas. Así, cuando " $L R$ " y " $L \neg R$ " carecen de valor de verdad, la ley de Raz no es verdadera. A su vez, también es posible probar que cuando " $L R$ " y " $L \neg R$ " carecen de valor de verdad, la ley de Raz no es falsa, entonces se sigue en $T L$

$$
\begin{aligned}
& (\neg T L R \& \neg T \neg L R \& \neg T L \neg R \& \neg T \neg L \neg R) \rightarrow \\
& {[\neg T(\neg L R \leftrightarrow L \neg R) \& \neg T \neg(\neg L R \leftrightarrow L \neg R)] .}
\end{aligned}
$$

${ }^{25}$ Acerca de la equivalencia entre $T(p \vee q)$ y $T p \vee T q$, véase von Wright 1984 c, p. 27. 
Esto muestra con claridad que la intuición central de Raz acerca de la existencia de lagunas ordinarias lleva a rechazar, en $T L$, que su tesis central $(\neg L R \leftrightarrow L \neg R)$ sea verdadera. Más bien, lo que la fórmula (14) pone de manifiesto es que cuando se admite que " $L R$ " y " $L \neg R$ " carecen de valor de verdad, entonces también hay que admitir que la ley de Raz no es verdadera ni falsa.

Sin embargo, podría sostenerse que la ley de Raz podría sobrevivir a una reconstrucción más cuidadosa en $T L$. Así, las intuiciones principales de Raz deberían ser reformuladas de otra manera a efectos de preservar su alcance en $T L$. Esta reformulación será nuestro objetivo en la siguiente sección.

\section{La ley de Raz: dos interpretaciones}

Hemos visto que es necesario encontrar una expresión que desempeñe un papel similar a la ley de Raz en $T L$. Por una parte, la nueva versión de la ley de Raz debe rechazar la existencia de lagunas genuinas. De otro modo, una de las ideas más importantes de Raz - que no existen lagunas cuando el derecho guarda silencio - sería abandonada. Por otra parte, debemos preservar aquellos teoremas que se derivan de su tesis (4) ya que, en caso contrario, en nuestra reconstrucción se perdería parte de la información relevante lógicamente vinculada con la ley de Raz.

En esta sección analizaremos dos posibles interpretaciones de la ley de Raz. Ambas versiones están parcialmente confundidas en la ambigüedad de la expresión " $\neg L R$ " que puede ser entendida como si dijera no sólo que una proposición jurídica no es verdadera, sino también que es falsa. De esta manera, la ley de Raz podría representarse de dos maneras diferentes:

(4a) $\neg T L R \leftrightarrow T L \neg R, \mathrm{y}$

(4b) $T \neg L R \leftrightarrow T L \neg R$

A fin de analizar claramente los compromisos conceptuales que se derivan de ambas tesis agregaremos (4a) o (4b) a $T L$ para explorar sus consecuencias. No intentaremos mostrar de qué manera ellas pueden ser justificadas en este cálculo. Apoyándonos 
en esta suposición intentaremos responder a las siguientes preguntas:

¿Estas interpretaciones de la ley de Raz rechazan la existencia de lagunas genuinas?

¿Estas interpretaciones de la ley de Raz rechazan la existencia de lagunas ordinarias? En otras palabras, ¿implican estas interpretaciones el principio de bivalencia?

¿Implican estas interpretaciones de la ley de Raz la tesis de las respuestas jurídicas completas?

\section{A. La primera interpretación}

Nuestro primer candidato dice: "No es verdad que jurídicamente exista una razón concluyente si y sólo si es verdad que jurídicamente no hay una razón concluyente..."

En símbolos:

(4a) $\neg T L R \leftrightarrow T L \neg R$

Esta fórmula tiene las siguientes características (véase el apéndice 1, al final de este artículo):

(i) implica el rechazo de las lagunas ordinarias;

(ii) implica el rechazo de las lagunas genuinas, y

(iii) implica la tesis de las respuestas completas.

B. La segunda interpretación ${ }^{26}$

Nuestro segundo candidato afirma: "Es falso que jurídicamente exista una razón concluyente... si y sólo si es verdad que jurídicamente no hay una razón concluyente. . "

En símbolos:

(4b) $T \neg L R \leftrightarrow T L \neg R$

Esta fórmula tiene las siguientes características (véase el apéndice 2, al final de este texto):

${ }^{26}$ En nuestro análisis supondremos y no trataremos de probar la consistencia de las lagunas ordinarias y $T L$. De igual manera, se supone sin prueba que (4b) y $T L$ son consistentes. Estamos agradecidos con uno de los evaluadores anónimos de Crítica por señalarnos este problema y en qué condiciones se sostienen las conclusiones que obtenemos en nuestro trabajo. 
(i) es implicada por la existencia de lagunas ordinarias,

(ii) implica el rechazo de las lagunas genuinas, y

(iii) no implica la tesis de las respuestas completas.

A primera vista, la única diferencia entre (4a) y (4b) está a la izquierda de ambos bicondicionales. En el primer caso tenemos “ $\neg T p$ ", mientras que en el segundo tenemos " $T \neg p$ ". En $T L$, " $T \neg p$ " implica " $\neg T p$ ". Por tanto, ¿no podría ser esto un indicio de que (4b) implica (4a)? La respuesta es negativa. Aunque el axioma (Al) $T \neg p \rightarrow \neg T p$ asegura que la falsedad de una proposición implica que ella no es verdadera, no puede probarse que (4b) $T \neg L R \leftrightarrow T L \neg R$ implique (4a) $\neg T L R \leftrightarrow T L \neg R$. La falta de implicación parece clara: mientras que (4a) implica bivalencia, (4b) está implicada por el rechazo de la bivalencia. Por tanto, si (4a) estuviese implicada por (4b), se seguiría por transitividad que el principio de bivalencia podría derivarse de su negación.

La existencia de lagunas ordinarias implica (4b) y la negación de (4a), es decir, tanto "( $T \neg L R \leftrightarrow T L \neg R)$ " como “ $\neg(\neg T L R \leftrightarrow T L \neg R)$ ". Esta última negación es equivalente a " $T L R \leftrightarrow T L \neg R$ ", y por ello es fácil mostrar que " $T L R \leftrightarrow$ $T \neg L R$ " es una consecuencia de las lagunas ordinarias. Pero, ¿no es una contradicción sostener que una proposición es verdadera si y sólo si es falsa? La respuesta es negativa. La fórmula ( $T L R \leftrightarrow T \neg L R$ ) es equivalente a aceptar que " $L R$ " no es verdadera ni falsa.

La fórmula ( $T L R \leftrightarrow T \neg L R)$ es equivalente a (15) $(T T L R \& T T \neg L R) \vee(T \neg T L R \& T \neg T \neg L R)$.

De (15) podemos derivar:

(a) $(T L R \& T \neg L R) \vee(\neg T L R \& \neg T \neg L R)$ A5

(b) $\neg(T L R \& T \neg L R) \rightarrow(\neg T L R \& \neg T \neg L R)$

a, $L P$

(c) $(\neg T L R \vee \neg T \neg L R) \rightarrow(\neg T L R \& \neg T \neg L R)$

$\mathrm{b}, L P$

(d) $(T L R \vee T \neg L R) \rightarrow(T L R \& T \neg L R)$

Contraposición en c, $L P$

El consecuente de (d) es refutable en $T L$, y por ello tenemos, modo tollente, $\neg(T L R \vee T \neg L R)$, que es equivalente 
a $(\neg T L R \& \neg T \neg L R)$. Por consiguiente, $T L R \leftrightarrow T \neg L R$ sólo conduce a aceptar que " $L R$ " no es verdadera ni falsa. Es interesante señalar que (d) presenta una estructura similar a la de una proposición antinómica. Según von Wright, la proposición $p$ es antinómica si y sólo si se puede probar la fórmula $(T p \vee T \neg p) \rightarrow(T p \& T \neg p) .{ }^{27}$ De esta fórmula, von Wright señala lo siguiente:

La conjunción de $(T p \& T \neg p)$ implica la conjunción de $(\neg T p \&$ $\neg T \neg p)$. Así, por transitividad, tenemos $(T p \vee T \neg p) \rightarrow(\neg T p \& \neg T$ $\neg p)$. El consecuente de esta implicación es la negación del antecedente. De allí, por el principio que los escolásticos denominaron consequentia mirabilis, la implicación es equivalente a su consecuente. Así, tenemos $(\neg T p \& \neg T \neg p)$. Por consiguiente, si el razonamiento en un sistema obedece las leyes de $T L$, la aparición de una proposición antinómica en el sistema significa que esa proposición carece de valor de verdad, no es verdadera ni falsa. ${ }^{28}$

De esta manera, las antinomias están estrechamente ligadas al rechazo de la bivalencia ya que, aunque no toda proposición que carece de valor de verdad es antinómica, las proposiciones antinómicas necesariamente carecen de valor de verdad.

\section{Conclusiones}

Una importante novedad de la propuesta de Raz es el rechazo del principio de bivalencia en el análisis del discurso acerca del derecho. Sin embargo, esta propuesta se ve oscurecida por una importante ambigüedad en su simbolismo. En particular, hemos considerado dos interpretaciones de la denominada "ley de Raz". La primera interpretación, (4a) $\neg T L R \leftrightarrow T L \neg R$, implica el principio de bivalencia, y por ello es incapaz de dar cuenta de lagunas ordinarias. Por el contrario, la segunda interpretación, (4b) $T \neg L R \leftrightarrow T L \neg R$, no implica bivalencia, y parece recoger mejor las intuiciones originales de Raz.

La propuesta original de $\operatorname{Raz}, \neg L R \leftrightarrow L \neg R$, tiene las siguientes consecuencias: (a) rechaza las lagunas genuinas; y (b) im-

27 Von Wright 1996, p. 84.

28 Von Wright 1996, p. 84. 
plica la tesis de las respuestas completas. Hemos visto que Raz no analiza expresamente la compatibilidad de su propuesta, $\neg L R \leftrightarrow L \neg R$, con la existencia de lagunas ordinarias. Esto oculta cierta tensión en su propuesta, ya que si la ley de Raz implica la tesis de las respuestas completas y esta tesis implica la negación de las lagunas ordinarias, se sigue que la verdad de la tesis central de Raz también niega las lagunas ordinarias. Sin embargo, esta conclusión es incompatible con la afirmación de Raz de que estas lagunas son inevitables. Esta dificultad se hace evidente en las interpretaciones que hemos analizado en $T L$.

En el siguiente cuadro se resumen las principales características de ambas interpretaciones:

\begin{tabular}{|l|c|c|c|}
\hline $\begin{array}{l}\text { Tesis 4 (ley de Raz): } \\
\text { interpretaciones }\end{array}$ & $\begin{array}{l}\text { ¿Rechaza } \\
\text { las lagunas } \\
\text { genuinas? }\end{array}$ & $\begin{array}{l}\text { ¿Es compatible } \\
\text { con las lagunas } \\
\text { ordinarias? }\end{array}$ & $\begin{array}{l}\text { ¿Implica la tesis } \\
\text { de las respues- } \\
\text { tas completas? }\end{array}$ \\
\hline 4) $\neg L R \leftrightarrow L \neg R$ & Sí & ¿No? & Sí \\
\hline 4a) $\neg T L R \leftrightarrow T L \neg R$ & Sí & No & Sí \\
\hline 4b) $T \neg L R \leftrightarrow T L \neg R$ & Sí & Sí & No \\
\hline
\end{tabular}

Este cuadro señala claramente las dificultades para articular todas las intuiciones relevantes del enfoque de Raz. En particular, el contenido conceptual de la presentación original de Raz, i.e., lo que Raz afirma expresamente más todas sus consecuencias lógicas, no puede ser capturado por ninguna de las formulaciones alternativas en $T L$. Es obvio que la interpretación (4b) reproduce con mayor fidelidad sus intuiciones explícitamente formuladas y por ello debe ser considerada la mejor candidata, en $T L$, para dar cuenta de las tesis de Raz acerca de las lagunas del derecho.

Finalmente, es necesario señalar que hay una importante intuición de Raz que no se puede preservar en $T L$. Según Raz, ${ }^{29}$

Un criterio de adecuación de cualquier enfoque de la vaguedad es que pueda reconocer como caso central de ésta aquellos casos en los que la vaguedad es "continua".

Los términos son vagos en este sentido si, además de los casos que quedan en la frontera entre el área cubierta por el concepto

${ }^{29}$ LSG, p. 73. 
y la que éste no cubre, hay casos límite entre aquellos cubiertos por el término y aquellos que pertenecen a la frontera; de igual modo existen casos límite entre aquellos a los cuales el término no se aplica y aquellos de la frontera, y así indefinidamente.

Por esta razón, Raz sugiere, en una nota, que su enfoque de la vaguedad podría conducir a una semántica que aceptase "infinitos números de valores de verdad". 30 Debe señalarse que este problema de la vaguedad de "segundo orden" no ha recibido una respuesta que cuente con consenso mayoritario en los análisis contemporáneos. El cálculo $T L$ no puede, al menos en la presentación que hemos analizado en este trabajo, dar cuenta de este tipo de vaguedad. Ello es así porque las $T$-fórmulas son siempre verdaderas o falsas. En este sentido, von Wright señala que $T L$, tal cual ha sido presentada, no puede resolver este problema ya que: ${ }^{31}$

la fórmula $\neg T T p \& \neg T \neg T p$ es refutable, "lógicamente falsa". Pero es perfectamente posible construir una lógica de la verdad $T L$ en la cual no sea así, i.e., en la cual la ley del tercero excluido no se mantenga ni siquiera en la forma muy débil que dice que toda proposición (individual) es verdadera o no es verdadera.

Una $T L$ capaz de dar cuenta del problema de la vaguedad de segundo orden tendría que abandonar la regla de la verdad, es decir, la regla de inferencia R3 del cálculo $T L$, y el metateorema M1. ${ }^{32}$ En caso contrario, la "lógica $T L$ de último grado" que se construya se comportará de manera clásica. Por esta razón, von Wright concluye: ${ }^{33}$

Podría también expresarse esta idea diciendo que las diversas lógicas de la verdad (truth-logics) se sistematizan (se estudian como lógicas) desde un punto de vista clásico. ¿Debe la lógica bivalente

30 LSG, p. 74, nota 18.

31 Von Wright 1984b, p. 113.

32 Von Wright 1996, p. 89.

33 Von Wright 1996, p. 90. 
clásica mantener esta "posición privilegiada" en un estudio sistemático de todas las lógicas? No intentaré responder aquí esta pregunta.

Al igual que von Wright, no intentaremos resolver aquí el desafío que significa reconstruir las tesis de Raz mediante una lógica que admita, como Raz señala, "infinitos números de valores de verdad". La respuesta satisfactoria a este desafío deberá aguardar hasta otro trabajo.

Apéndice 1: La tesis (4a) $\neg T L R \leftrightarrow T L \neg R$

Puede mostrarse que esta fórmula (i) implica el rechazo de las lagunas genuinas; (ii) implica el rechazo de las lagunas ordinarias, y (iii) implica la tesis de las respuestas completas.

A) Lagunas genuinas. (4a) implica el rechazo de las lagunas genuinas. Prueba:

a) $\neg T L R \leftrightarrow T L \neg R$

b) $T(\neg L R \& \neg L \neg R)$

c) $T \neg L R \& T \neg L \neg R$

Ley de Raz en $T L$

d) $\neg T L R \& T \neg L \neg R$

Lagunas genuinas

$\mathrm{b}, \mathrm{A} 3$

e) $\neg T L R$

f) $T L \neg R$

c, Al, $L P$

h) $T \neg L \neg R$

i) $\quad T \neg L \neg R \& T L \neg R$

Eliminación en d

a, e, $M P$

Eliminación en d

f, h

Dado que en $T L$ no se admite que una proposición, i.e., " $L \neg R$ ", sea verdadera y falsa, (4a) y la hipótesis acerca de la existencia de lagunas genuinas son contradictorias. Por ello, esta nueva versión de la ley de Raz rechaza la existencia de lagunas genuinas en el derecho.

B) Lagunas ordinarias. (4a) también es incompatible con las lagunas ordinarias, i.e., cuando ambas " $L R$ " y " $L \neg R$ " carecen de valores de verdad. Prueba:
a) $\neg T L R \& \neg T \neg L R \& \neg T L \neg R \&$
Laguna ordinaria
$\neg T \neg L \neg R$
b) $\neg T L R \leftrightarrow T L \neg R$
4a
c) $\neg T L R$
d) $T L \neg R$
Eliminación en a
e) $\neg T L \neg R$
b, c, $M P$
Eliminación en a 
f) $\quad T L \neg R \& \neg T L \neg R$

d, e

Dado que (f) es refutable en $T L$, la existencia de lagunas genuinas implica el rechazo de (4a).

C) La ley de Raz y la tesis de las respuestas completas. A partir de (4a) podemos probar la tesis de las respuestas completas en $T L$.
a) $\neg T L R \leftrightarrow T L \neg R$
(4a)
b) $\neg T L R \rightarrow T L \neg R$
Eliminación en a
c) $\quad T L R \vee T L \neg R$
b, $L P$
d) $\quad T(L R \vee L \neg R)$
A3, A4

Apéndice 2: la tesis (4b) $T \neg L R \leftrightarrow T L \neg R$

Puede mostrarse que esta fórmula (i) está implicada por la existencia de lagunas ordinarias, (ii) implica el rechazo de las lagunas genuinas, y (iii) no implica la tesis de las respuestas completas.

A) Lagunas genuinas. (4b) implica la negación de las lagunas genuinas. Prueba:
a) $T \neg L R \leftrightarrow T L \neg R$
$4 \mathrm{~b}$
b) $\quad T(\neg L R \& \neg L \neg R)$
Lagunas genuinas
c) $T \neg L R \& T \neg L \neg R$
b, A3
d) $T \neg L R$
Eliminación en c
e) $T L \neg R$
f) $T \neg L \neg R$
a, d, $M P$
g) $T L \neg R \& T \neg L \neg R$
Eliminación en c
e, f.

Dado que (g) es refutable en $T L$, debemos admitir que la aceptación de (4b) compromete a rechazar la existencia de lagunas genuinas.

B) Lagunas ordinarias. A diferencia de (4a), (4b) no implica la bivalencia. Por el contrario, (4b) es implicada por la presencia de una laguna ordinaria. Esta conclusión puede probarse mediante la siguiente reductio ad absurdum.
a) $\neg T L R \& \neg T \neg L R \& \neg T L \neg R \&$
Lagunas ordinarias
$\neg T \neg L \neg R$
b) $\neg(T L \neg R \leftrightarrow T \neg L R)$
Hipótesis
c) $T L \neg R \leftrightarrow \neg T \neg L R$
b, $L P$
d) $\neg T \neg L R$
Eliminación en a 
e) $T L \neg R$

f) $\neg T L \neg R$

g) $\quad T L \neg R \& \neg T L \neg R$

h) $\neg \neg(T L \neg R \leftrightarrow T \neg L R)$

i) $\quad T \neg \neg(T L \neg R \leftrightarrow T \neg L R)$

j) $\quad T(T L \neg R \leftrightarrow T \neg L R)$ c, d, $M P$

Eliminación en a

$\mathrm{e}, \mathrm{f}$

$\mathrm{g}$, reductio ad absurdum

Regla de la verdad

i, A2

Por consiguiente, la existencia de lagunas ordinarias implica que $(4 \mathrm{~b})$ es verdadera.

C) La ley de Raz y la tesis de las respuestas jurídicas completas. La tesis de las respuestas completas no puede derivarse de (4b). De otro modo, (4b) y la existencia de lagunas ordinarias llevarían a una contradicción. Prueba:
a) $\quad \neg T L R \& \neg T \neg L R \& \neg T L \neg R \& \quad$ Lagunas ordinarias $\neg T \neg L \neg R$
b) $\quad(T \neg L R \leftrightarrow T L \neg R) \rightarrow$ $T(L R \vee L \neg R)$
Hipótesis
c) $(T \neg L R \leftrightarrow T L \neg R)$
$4 \mathrm{~b}$
d) $\quad T(L R \vee L \neg R)$
$\mathrm{b}, \mathrm{c}, M P$
e) $\quad T L R \vee T L \neg R$
$L P, \mathrm{~A} 4 \mathrm{~d})$
f) $\neg T L R$
a, $L P$
g) $T L \neg R$
e, f, $L P$
h) $\neg T L \neg R$
a, $L P$
i) $T L \neg R \& \neg T L \neg R$
$\mathrm{g}, \mathrm{h}$

La conjunción de (a) y (e) es una contradicción. Así, el conjunto $\{b, c\}$ implica bivalencia, pero la bivalencia y las lagunas ordinarias son obviamente incompatibles. Por ello, para superar esta incoherencia es necesario rechazar que existan lagunas ordinarias, o bien, si no, nuestra hipótesis (b), i.e., que la tesis de las respuestas jurídicas completas está implicada por (4b).

\section{BIBLIOGRAFÍA}

Alchourrón, Carlos y Eugenio Bulygin (1971), Normative Systems, Springer Verlag, Viena/Nueva York.

Bix, Brian (1993), Law, Language and Legal Determinacy, Oxford University Press, Oxford.

Eisenberg, Melvin Aron (1988), The Nature of the Common Law, Harvard University Press, Cambridge, Mass. 
Gardner, John (1988), "Concerning Permissive Sources and Gaps", Oxford Journal of Legal Studies, no. 8, pp. 457-461.

Raz, Joseph (1979) [LSG], "Legal Reasons, Sources and Gaps", The Authority of Law, Oxford University Press, Oxford, pp. 53-77.

Shiner, Roger (1992), Norm and Nature, Oxford University Press, Oxford.

Von Wright, G.H. (1996), "Truth-Logics", Six Essays in Philosophical Logic, Acta Philosophica Fennica, vol. 60, Philosophical Society of Finland, Helsinki, pp. 71-91.

(1984), Truth, Knowledge and Modality, Basil Blackwell, Oxford.

L (1984a), "Demystifying Propositions", Truth, Knowledge and Modality, pp. 14-25.

— (1984b), "Logical Modality", Truth, Knowledge and Modality, pp. 104-116.

— (1984c), "Truth and Logic", Truth, Knowledge and Modality, pp. 26-41.

Recibido el 20 de noviembre de 1999; revisado el 22 de octubre de 2001; aceptado el 7 de noviembre de 2001 